\title{
Adherencia en heroinómanos: la potencia predictiva de los estadios de cambio evaluados durante la admisión al tratamiento
}

\author{
Albiach Catalá, C.*; llinares Pellicer, M.C.**; Palau Muñoz, C.***; Santos Diez, P.**** \\ * Psicóloga. Unidad de Conductas Adictivas de la Mancomunitat L'Horta Nord, Massamagrell, Valencia. \\ * Psicóloga. Unidad de Conductas Adictivas de la Mancomunitat de Municipis de la Safor, Gandia, Valencia. \\ *** Psicóloga. Unidad de Conductas Adictivas del Área de Salud n 5 . Paterna Valencia. Consellería de Sanidad. Generalitat Valenciana. \\ **** Dr. en Psicología. Unidad de Conductas Adictivas del Área de Salud no 13. Xàtiva. Valencia. Consellería de Sanidad. Generalitat Valenciana.
}

Patxi Santos Diez, U.C.A. Xàtiva. Hospital Antiguo. 46800. Xàtiva. Valencia. Correo Electrónico: patxisan@correo.cop.es

\section{Resumen}

Tomando como marco de referencia el modelo transteórico de cambio de Prochaska y DiClemente, el objetivo del presente estudio es evaluar el estadio de cambio en el que se ubican 317 heroinómanos (250 varones y 67 mujeres), utilizando una entrevista semiestructurada. Además, se estudia el poder predictivo del estadio de cambio, las variables sociodemográficas, toxicológicas y los tipos de tratamiento relacionados con la adherencia al mes, tres, seis, nueve y doce meses.

Los resultados obtenidos indican que el estadio de cambio no es una potente variable predictora de la adherencia al tratamiento, ya que solamente se muestra estadísticamente significativa al mes y a los 3 meses; otras variables estudiadas, sin embargo, sí predicen adherencia durante todos los cortes temporales medidos: edad y tipo de tratamiento que inicia. Por otra parte, y en menor medida, los tratamientos anteriores, el tiempo máximo de abstinencia previa y el tipo de acompañamiento, tienden a predecir adherencia en los últimos cortes temporales.

Palabras clave: estadio de cambio, adherencia, heroína, variables predictoras.

\section{INTRODUCCIÓN}

s una realidad que a lo largo de los últimos años se ha avanzado mucho en el conocimiento del fenómeno de las drogodependencias. Concretamente en el ámbito de la asistencia, hemos asistido a una diversificación de los tratamientos, con nuevos fármacos, nuevos programas psicoterapéuticos, etc. que ha permitido poder ofertar una mayor diversidad de tratamientos atendiendo a las necesidades individuales de cada sujeto heroinómano. Estos avances, a pesar de haber producido un cambio sustancial en los resultados terapéuticos, no lo han hecho en la medida en que cabía esperar.

\section{Abstract}

Taking the transtheorical model of change of Prochaska and DiClemente as a reference, the aim of this study is to evaluate the stage of change in which are located 317 heroin addicts (250 men and 67 women), using a semi-estructurate interview. Also, it studies the predictive power of the stage of change, the social-demographic and toxicologic variables and types of treatment related to the adherence, measured a month, at three months, at six months, at nine months and twelve months.

The results obtained show that the stage of change is not a potent predictive variable of the adherence to treatment, since it is only stadistically significative at a month and at 3 months. Other estudied variables predict adherence in all temporal points measured: age and type of treatment that begins. Previous treatments, maximum time of previous abstinence and the type of companionship tends to predict adherence in the last temporary points.

Key words: stage of change, adherence, heroine, predictive variables.

Existe una abundante evidencia clínica de que el abandono precoz de un tratamiento por adicción representa en la mayoría de los casos, la recaída en el abuso de drogas, con todas las consecuencias negativas que ello conlleva (Caballero, 1988). Otros estudios (Tucker et al., 1985; Secades et al. 1991), sugieren que los pacientes que obtienen el alta terapéutica tienen menos probabilidad de recaer que los sujetos que no finalizan el tratamiento. Vaillant (1973) y Hubbard et al. (1984) encontraron una relación inversa entre la duración de la estancia en el programa y la recaída posterior. De hecho, el tiempo de permanencia en el programa es el predictor más consistente de un resultado exitoso (Kooyman, 1996). 
Revisando estudios sobre retención en tratamiento en sujetos heroinómanos donde se evalúa motivación y alianza terapéutica, hemos hallado el estudio de Simpson el al. (1997), en el que se evalúa la motivación previa al tratamiento y el rápido compromiso terapéutico en 435 heroinómanos. Los resultados indican que los pacientes que permanecen un año o más en tratamiento tienen cerca de cinco veces más posibilidades de tener mejores resultados post-tratamiento; así como que el mayor tiempo en tratamiento está precedido por una mayor motivación del paciente en la admisión y a un rápido establecimiento de la alianza y/o compromiso terapéutico en el programa.

El mayor tiempo de tratamiento está predicho por una mayor motivación del paciente en el momento de la admisión al mismo. Según nuestra experiencia profesional en este campo, hemos podido constatar cómo la mayoría de los sujetos que acuden a los servicios ambulatorios lo hacen presionados, cuando no obligados, por el entorno familiar más próximo, existiendo en estos casos escasa conciencia de problemática cuando no negación de la misma.

\section{MODELOTRANSTEÓRICO DE CAMBIO DE PROCHASKA Y DICLEMENTE}

En el campo de las adicciones, existen diferentes modelos explicativos de cómo los sujetos modifican su comportamiento adictivo. Quizás de todos ellos, el que estudia de forma más global y diferenciada el curso del proceso de cambio es el Modelo Transteórico de Prochaska y DiClemente. Es éste un modelo tridimensional que integra estadios, procesos y niveles de cambio (Prochaska et al., 1992a). Según este modelo los estadios de cambio son los siguientes:

Precontemplación: En este estadio los individuos no piensan seriamente en cambiar, no creen tener problemas, al menos en la misma medida que lo hacen otros que están en estadios más avanzados, y la posibilidad de acudir a tratamiento es algo que ni siquiera han considerado.

Contemplación: En este caso los sujetos están pensando seriamente en el cambio a lo largo de los próximos seis meses, tienen un mayor nivel de conciencia del problema, han considerado la posibilidad de cambiar. Es un período de ambivalencia en el que coexisten sentimientos contradictorios, sobre continuar con la adicción o dejarla.

Preparación: Esta situación representa tanto determinación, como preparación para iniciar un tratamiento, por lo que se presupone que el individuo realizará un intento serio por cambiar en un futuro inmediato.

Actuación: Se produce una modificación manifiesta de la conducta problemática incluida la abstinen-
cia.Mantenimiento: Se caracteriza por el mantenimiento y generalización de la abstinencia a otras drogas así como la consolidación de un nuevo estilo de vida.

Recaída: Ocurre cuando las estrategias de cambio utilizadas fracasan en estabilizar al individuo en los estadios de acción o mantenimiento, provocando una vuelta hacia estadios previos, frecuentemente al de contemplación o al de preparación, y en el peor de los casos al de precontemplación.

Finalización: Cuando se consiguen estabilizar los cambios conductuales mas allá de la fase de mantenimiento se especula sobre un posible estadio de finalización, definido como la no existencia de tentaciones a través de todas las situaciones problema, es decir, la extinción absoluta de la conducta adictiva sin la necesidad de la utilización de procesos de cambio para el mantenimiento de los nuevos patrones de comportamiento.

Los procesos de cambio hacen referencia a la actividad iniciada o experimentada por un individuo, que modifica el efecto, la conducta, las cogniciones o las relaciones interpersonales y los niveles de cambio representan la una organización jerárquica, en cinco niveles distintos e interrelacionados, de los problemas psicológicos susceptibles de ser tratados (Tejero y Trujols, 1994). Tradicionalmente cada sistema psicoterapéutico ha atribuido los problemas psicológicos a uno o dos de estos niveles centrando su atención en la resolución de los mismos. Estos niveles son:

- Síntoma / situación.

- Cogniciones desadaptativas

- Conflictos actuales interpersonales

- Conflictos de familia / sistemas

- Conflictos intrapersonales.

Estos autores (DiClemente y Prochaska, 1995) en un estudio con fumadores observaron que entre un $10-15 \%$ se encontraban preparados para la acción, entre un 30-40\% en el estadio de contemplación y entre un 50-60\% en el estadio de pre-contemplación. Otros autores estadounidenses (Vellicer et cols., 1995) encuentran resultados similares. En España, Becoña y cols. (1994), encuentran proporciones superiores en el estadio de precontemplación (68\%), inferiores en contemplación (25\%) y preparación (7\%).

En cuanto al poder predictivo de los estadios de cambio en fumadores, un estudio de Farkas, et cols (1996) encontró que los estadios de cambio no eran un predictor significativo del cese de la conducta adictiva; sin embargo, la ecuación que combina fumar ocasional/diariamente, $\mathrm{n}^{\circ}$ de cigarrillos diarios, periodos de abstinencia previa, periodos de abstinencia superiores a cinco días, discriminaron la abstinencia de 1 a 2 años mejor que los estadios de cambio. Sin embargo, varios autores han criticado por diversos motivos 
metodológicos y conceptuales este estudio (Prochaska y Velicer, 1996).

La utilización del modelo transteórico de Prochaska y DiClemente en el caso del trastorno por dependencia a opiáceos aún es, en la actualidad, limitada. Unicamente tres trabajo publicados (Abellanas y Mc. Lellan, 1993; Insenhart 1994, y Cusker et al 1996) abordan la utilidad de la dimensión de los estadios de cambio en muestras de adictos a opiáceos, no evaluándose en ninguno de ellos la potencial validez y utilidad terapéutica de la dimensión procesos de cambio.

Según nuestros propios datos de un estudio preliminar con una muestra de heroinómanos, encontramos el $17.2 \%$ de precontempladores y el $70.5 \%$ contempladores (Albiach, C. y cols., 1996). Según Prochaska et al. (1992b) las terapias orientadas a la acción pueden ser bastantes eficaces en sujetos que se encuentran en el estadio de preparación o actuación, pero estos mismos programas pueden ser ineficaces o insuficientes en sujetos en el estadio de precontemplación y en algunos contempladores (y recordemos que estadísticamente son los más numerosos). De ahí la necesidad de evaluar los estadios de cambio en el momento de la admisión como paso previo a una adecuada selección del tratamiento.

Debido a la influencia que la retención de los pacientes tiene sobre la eficacia del tratamiento, se han venido realizando numerosos estudios que pretenden delimitar qué variables predictoras determinan la mayor o menor adherencia de los adictos en los diferentes programas terapéuticos. Desde esta perspectiva, pretendemos profundizar en el estudio de dichas variables predictoras.

\section{OBJETIVOS.}

- Estudiar la evolución de la adherencia al tratamiento de los sujetos adictos a opiáceos a lo largo de un año, relacionándola con las variables sociodemográficas y toxicológicas.

- Conocer la capacidad predictiva del estadio de cambio en que se ubican los sujetos cuando acuden a Centros Ambulatorios sobre la retención en los diferentes programas terapéuticos.

\section{MATERIAL Y MÉTODO.}

DISEÑO.

Estudio longitudinal y multicéntrico de seguimiento sobre usuarios heroinómanos que demandaron asistencia en centros ambulatorios para el tratamiento de las adicciones en el periodo de tiempo comprendido entre Febrero de 1995 y Septiembre de 1997. El periodo de seguimiento de la retención es de dos años, siendo los datos que se presentan en este estudio los correspondientes a los doce primeros meses.

\section{SUJETOS.}

La muestra está constituida por 317 dependientes a la heroína (250 varones y 67 mujeres) que demandaron asistencia en tres servicios ambulatorios para el tratamiento de las adicciones de la provincia de Valencia: Unidad de Conductas Adictivas (UCA) de la Mancomunitat de L'Horta Nord en Massamagrell, UCA de la Cruz Roja de Valencia y UCA de la Mancomunitat de Minicipis de La Safor en Gandia. Se han incluido en este estudio todos los sujetos que solicitaron tratamiento y fueron atendidos al inicio por la psicóloga de los servicios (en la mayoría de los casos coincide con la acogida). Se han excluido los usuarios que presentaban comorbilidad psiquiátrica grave (psicosis, retraso mental o estado demencial).

\section{MATERIAL.}

Una entrevista semiestructurada de respuesta abierta elaborada por los autores y constituida por las siguientes cuatro preguntas abiertas: 1- ¿Cuál es el motivo por el que ha acudido al servicio? 2 - Cuáles son las razones por las que ha venido (en el caso de que no mencione abandono del consumo o problemática con la sustancia) o, para abandonar el consumo (si lo menciona como motivo para acudir al centro). 3¿En qué le podemos ayudar?. 4 - ¿Cuál es su situación respecto al consumo de drogas?. El análisis de las respuestas nos permite poder ubicarlos en el estadio de cambio correspondiente, siguiendo las directrices del modelo transteórico de cambio.

La entrevista constituye también el instrumento para la obtención de los datos relativos a las variables toxicológicas. Se registra la información aportada por el sujeto durante la primera entrevista psicológica pasando toda la información obtenida a formar parte de la historia clínica.

Los datos que conforman las variables demográficas se obtienen de la historia clínica; asimismo, se registra mediante observación el hecho de acudir al centro acompañado o no, y de quien.

Los datos han sido tratados con el paquete estadístico EPIINFO.

\section{PROCEDIMIENTO}

Durante la primera entrevista psicológica, de forma intercalada a otras cuestiones, procedemos a formular las cuatro preguntas abiertas que conforman la entrevista semiestructurada y el resto de cuestiones que conforman las variables toxicológicas y sociodemográficas. 
La información obtenida a partir de la entrevista semiestructurada nos permite analizar los aspectos tanto cognitivos como comportamentales: sus intenciones, a corto y largo plazo respecto a la abstinencia; el nivel de concienciación de su problemática y la vinculación que establece con el uso de drogas; sus deseos de cambiar y en qué sentido; los cambios iniciados y los conseguidos; los pros y contras para el cambio y su historia relativa al uso de drogas. Previamente, a partir de la conceptualización del modelo transteórico y de las características que configuran cada estadio de cambio, establecimos entre los autores, los criterios para ubicar a los sujetos en el estadio de cambio correspondiente. Durante la entrevista se fomentó la expresión espontánea de los sujetos, propiciando un clima de dialogo y confianza. Para minimizar el posible sesgo producido por la subjetividad del entrevistador, se realizó la tarea de ubicar a los sujetos en su estadio correspondiente por parte de los tres entrevistadores restantes.

La adherencia al tratamiento se mide basándose en continuidad, abandono, alta, readmisión y otros (prisión y defunción), en cinco momentos: al mes, a los tres, seis, nueve y doce meses.

\section{RESULTADOS \\ Análisis descriptivo:}

La muestra estudiada (317 sujetos) se compone de 250 Varones (78.9\%) y 67 Mujeres $(21.1 \%$ ), siendo la edad media de 27 años con una desviación estandar de 7 años. El $72.6 \%$ son solteros y el $77.3 \%$ tiene un nivel de estudios de EGB. En cuanto a la situación laboral, el $65.3 \%$ de la muestra están parados y en activo el $29.7 \%$. Son obreros cualificados el 31.2\%, no cualificados el $26.2 \%$ y sin profesión el 25.6\%. (Tabla I). Estos resultados similares a los de otros estudios (Arias et al., 1996; Gutierrez et al., 1998; Pastor el al., 1999).

Con relación a las variables toxicológicas (Tabla II) señalar que predomina la ausencia de antecedentes familiares de conductas adictivas (65.6\%), aunque se observan antecedentes familiares de consumo de heroína en el $17 \%$ de la muestra y de alcohol en el $15.8 \%$. Existe policonsumo en el $87.1 \%$ de los casos siendo dos el número mayoritario de otras drogas consumidas. El $30.1 \%$ de la muestra se encuentra en el intervalo de 5 a 9 años de consumo, le sigue el $27.2 \%$ en el intervalo de 10 a 14 años y el $23.4 \%$ en el 0 a 4 años; la frecuencia acumulada entre los tres primeros intervalos definidos es del $80.7 \%$, lo que supone que este porcentaje ha consumido heroína durante menos de 14 años. Este perfil no difiere significativamente del encontrado en otras muestras españolas: Ordoñez et al. (1995), Marina et al. (1996) y Fernández et al. (1996).

\section{TABLA I - CARACTERÍSTICAS SOCIODEMOGRÁFICAS}

\begin{tabular}{|c|c|c|c|}
\hline & VARIABLES & $\mathrm{N}$ & FREC $\%$ \\
\hline \multirow[t]{2}{*}{ SEXO } & Varones & 250 & 78.9 \\
\hline & Mujeres & 67 & 21.1 \\
\hline \multirow[t]{7}{*}{ EDAD } & $<18$ & 2 & 0.6 \\
\hline & $18-24$ & 66 & 21.5 \\
\hline & $25-31$ & 142 & 44.8 \\
\hline & $32-38$ & 74 & 23.3 \\
\hline & $39-45$ & 25 & 7.9 \\
\hline & $46-52$ & 6 & 1.9 \\
\hline & $>52$ & 2 & 0.6 \\
\hline \multirow[t]{4}{*}{ ESTADO CIVIL } & Casado/Convivencia & 63 & 19.9 \\
\hline & Separado & 20 & 6.3 \\
\hline & Soltero & 230 & 72.6 \\
\hline & Viudo & 4 & 1.3 \\
\hline \multirow[t]{4}{*}{ ESTUDIOS } & Sin Estudios & 10 & 3.2 \\
\hline & E.G.B. & 245 & 77.3 \\
\hline & B.U.P./ F.P. & 48 & 15.1 \\
\hline & Estudios Superiores & 14 & 4.4 \\
\hline \multirow[t]{7}{*}{ PROFESIÓN } & Estudiante & 3 & 0.9 \\
\hline & Obrero cualificado & 99 & 31.2 \\
\hline & Obrero no cualificado & 83 & 26.2 \\
\hline & Pequeño empresario & 14 & 4.4 \\
\hline & Profesión liberal & 18 & 5.7 \\
\hline & Sin profesión & 81 & 25.6 \\
\hline & Otros & 19 & 6.0 \\
\hline \multirow{5}{*}{$\begin{array}{l}\text { SITUACIÓN } \\
\text { LABORAL }\end{array}$} & Activo & 94 & 29.7 \\
\hline & Parado & 207 & 65.3 \\
\hline & Pensionista & 2 & 0.6 \\
\hline & Incapacitado & 12 & 3.8 \\
\hline & Otros & 2 & 0.6 \\
\hline
\end{tabular}

Para el $35,6 \%$ de los sujetos es el primer tratamiento que demandan mientras que el $64,4 \%$ han recibido tratamientos previos. El $83 \%$ del total de los sujetos han recibido menos de tres tratamientos. El $40.1 \%$ no ha mantenido ningún periodo de abstinencia previo, mientras que un $18.3 \%$ han conseguido más de un año y el resto (41.6\%) entre menos de 15 días y un año. La vía de administración más empleada es la fumada $(67.2 \%)$, seguida de la endovenosa $(27.8 \%)$.

El $65.3 \%$ de los sujetos acudieron al servicio acompañados, siendo la madre $(20,5 \%)$ y la pareja $(19.2 \%)$ las que lo hacen mayoritariamente; el $34.7 \%$ acudieron solos. Tabla III.

Respecto al estadio de cambio en el que se ubican los sujetos al inicio del tratamiento, señalar que el $67.2 \%$ de los sujetos se encuentran en contemplación; el $13.9 \%$ en precontemplación, y el mismo porcentaje en preparación para la acción. Tan solo el $4.4 \%$ en actuación y el $0.6 \%$ en mantenimiento. Así pues, son un porcentaje muy pequeño los sujetos que se 


\begin{tabular}{|c|c|c|c|}
\hline \multicolumn{4}{|c|}{ TABLA II - CARACTERÍSTICAS TOXICOLÓGICAS } \\
\hline & VARIABLES & $\mathrm{N}$ & FREC $\%$ \\
\hline \multirow[t]{7}{*}{ N OTRAS DROGAS } & 0 & 41 & 12.9 \\
\hline & 1 & 77 & 24.3 \\
\hline & 2 & 120 & 37.9 \\
\hline & 3 & 52 & 16.4 \\
\hline & 4 & 24 & 7.6 \\
\hline & 5 & 2 & 0.6 \\
\hline & 6 & 1 & 0.3 \\
\hline \multirow[t]{7}{*}{ AÑOS DE CONSUMO } & $0-4$ & 74 & 23.4 \\
\hline & $5-9$ & 95 & 30.1 \\
\hline & $10-14$ & 86 & 27.2 \\
\hline & $15-19$ & 48 & 15.2 \\
\hline & $20-24$ & 11 & 3.5 \\
\hline & $25-29$ & 1 & 0.3 \\
\hline & $30-34$ & 1 & 0.3 \\
\hline TRATAMIENTOS & 0 & 112 & 35.3 \\
\hline \multirow[t]{8}{*}{ PREVIOS } & 1 & 64 & 20.2 \\
\hline & 2 & 53 & 16.7 \\
\hline & 3 & 34 & 10.7 \\
\hline & 4 & 30 & 9.5 \\
\hline & 5 & 14 & 4.4 \\
\hline & 6 & 8 & 2.5 \\
\hline & 7 & 1 & 0.3 \\
\hline & 8 & 1 & 0.3 \\
\hline PERIODOS DE & Ninguno & 127 & 40.1 \\
\hline \multirow[t]{6}{*}{ ABSTINENCIA } & $<15$ días & 14 & 4.4 \\
\hline & 16 - 30 días & 18 & 5.7 \\
\hline & $1-3$ meses & 29 & 9.1 \\
\hline & $4-6$ meses & 36 & 11.4 \\
\hline & $7-12$ meses & 35 & 11.0 \\
\hline & > 1 año & 58 & 18.3 \\
\hline VÍA DE & Endovenosa & 88 & 27.8 \\
\hline \multirow[t]{2}{*}{ ADMINISTRACIÓN } & Esnifada & 16 & 5.0 \\
\hline & Fumada & 213 & 67.2 \\
\hline ANTECEDENTES & Alcohol & 50 & 15.8 \\
\hline FAMILIARES DE & Cocaína & 1 & 0.3 \\
\hline \multirow[t]{3}{*}{ ADICCIÓN } & Heroína & 56 & 17.6 \\
\hline & Juego & 2 & 0.6 \\
\hline & Ninguna & 208 & 65.6 \\
\hline
\end{tabular}

encuentran preparados para la acción o en actuación en el momento de acudir a un centro ambulatorio.

Referente a los programas de tratamiento encontramos que el $9.1 \%$ no llegan a iniciar tratamiento, el $15.1 \%$ desintoxicación (finalizada o no), el $45.1 \%$ entra en un programa de Metadona, el 17.7\% Programas de Antagonistas y el $12 \%$ Programas Libres de drogas. Tabla III.

Respecto a la tasa de adherencia encontramos que, al mes de la admisión, la mayoría de sujetos $(84.5 \%)$ continúa acudiendo a los servicios, produciéndose una disminución progresiva en la retención

\begin{tabular}{|c|c|c|c|}
\hline \multicolumn{4}{|c|}{$\begin{array}{l}\text { TABLA III - VARIABLES MOTIVACIONAL, DE } \\
\text { TRATAMIENTO Y DE ACOGIDA. }\end{array}$} \\
\hline & VARIABLES & $\mathrm{N}$ & FREC $\%$ \\
\hline ESTADIO DE & Precontemplación & 44 & 13.9 \\
\hline \multirow[t]{4}{*}{ CAMBIO } & Contemplación & 213 & 67.2 \\
\hline & Preparación & 44 & 13.9 \\
\hline & Actuación & 14 & 4.4 \\
\hline & Mantenimiento & 2 & 0.6 \\
\hline PROGRAMA DE & No Inicia & 29 & 9.1 \\
\hline \multirow[t]{5}{*}{ TRATAMIENTO } & Motivación & 3 & 0.9 \\
\hline & Metadona & 143 & 45.1 \\
\hline & Desintoxicación & 48 & 15.1 \\
\hline & Antagonistas & 56 & 17.7 \\
\hline & Libre de Drogas & 38 & 12.0 \\
\hline ACUDE A LA & Solo & 110 & 34.7 \\
\hline \multirow[t]{8}{*}{ UNIDAD } & Amigo & 6 & 1.9 \\
\hline & Familia & 25 & 7.9 \\
\hline & Hermano & 15 & 4.7 \\
\hline & Madre & 65 & 20.5 \\
\hline & Otros & 11 & 3.5 \\
\hline & Padre & 19 & 6.0 \\
\hline & Padres & 5 & 1.6 \\
\hline & Pareja & 61 & 19.2 \\
\hline
\end{tabular}

\begin{tabular}{|c|c|c|c|}
\hline \multicolumn{4}{|c|}{ TABLA IV - ADHERENCIA AL TRATAMIENTO } \\
\hline & VARIABLES & $\mathrm{N}$ & FREC $\%$ \\
\hline \multirow{4}{*}{$\begin{array}{l}\text { ADHERENCIA AL } \\
\text { MES }\end{array}$} & Abandono & 41 & 12.9 \\
\hline & Continua & 268 & 84.5 \\
\hline & Derivación & 3 & 0.9 \\
\hline & Otros & 5 & 1.6 \\
\hline \multirow{4}{*}{$\begin{array}{l}\text { ADHERENCIA A LOS } \\
3 \text { MESES }\end{array}$} & Abandono & 74 & 23.3 \\
\hline & Continua & 232 & 73.2 \\
\hline & Derivación & 6 & 1.9 \\
\hline & Otros & 5 & 1.6 \\
\hline \multirow{4}{*}{$\begin{array}{l}\text { ADHERENCIA A LOS } \\
6 \text { MESES }\end{array}$} & Abandono & 116 & 36.8 \\
\hline & Continua & 186 & 59.0 \\
\hline & Derivación & 8 & 2.5 \\
\hline & Otros & 5 & 1.6 \\
\hline \multirow{5}{*}{$\begin{array}{l}\text { ADHERENCIA A LOS } \\
9 \text { MESES }\end{array}$} & Abandono & 128 & 37.8 \\
\hline & Continua & 172 & 59.0 \\
\hline & Derivación & 8 & 2.5 \\
\hline & Otros & 5 & 0.6 \\
\hline & Readmisión & 4 & 1.3 \\
\hline \multirow{5}{*}{$\begin{array}{l}\text { ADHERENCIA A LOS } \\
12 \text { MESES }\end{array}$} & Abandono & 133 & 41.9 \\
\hline & Continua & 155 & 48.9 \\
\hline & Derivación & 11 & 3.5 \\
\hline & Otros & 7 & 2.2 \\
\hline & Readmisión & 11 & 3.5 \\
\hline
\end{tabular}




\begin{tabular}{|c|c|c|c|c|c|}
\hline \multicolumn{6}{|c|}{$\begin{array}{l}\text { TABLA V - CORRELACIONES ENTRE LAS VARIABLES ESTUDIADASY LA ADHERENCIA } \\
\text { ALTRATAMIENTO. }\end{array}$} \\
\hline VARIABLES & $1 \mathrm{MES}$ & 3 MESES & 6 MESES & 9 MESES & 12 MESES \\
\hline Edad & $0.000 * * *$ & $0.000 * * *$ & $0.000 * * *$ & $0.000 * * *$ & $0.000 * * *$ \\
\hline Tratamientos ant. & 0.058 & 0.056 & $0.019 *$ & $0.019 *$ & $0.044 *$ \\
\hline Acompañado por... & 0.050 & 0.239 & 0.100 & $0.019 *$ & $0.000 * * *$ \\
\hline Estadio de Cambio & $0.003 * *$ & $0.001 * *$ & 0.113 & 0.511 & 0.067 \\
\hline Tipo de Programa & $0.000 * * *$ & $0.000 * * *$ & $0.000 * * *$ & $0.000 * * *$ & $0.000 * * *$ \\
\hline Adherencia al mes & $0.000 * * *$ & $0.000 * * *$ & $0.000 * * *$ & $0.000 * * *$ & $0.000 * * *$ \\
\hline \multicolumn{6}{|l|}{ 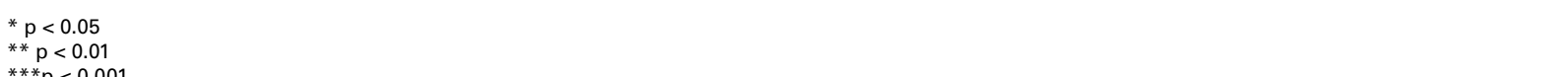 } \\
\hline
\end{tabular}

\begin{tabular}{|c|c|c|c|c|c|c|c|}
\hline \multicolumn{8}{|c|}{ TABLA VI } \\
\hline $\begin{array}{l}\text { Variable } \\
\text { PROGRAMA } \\
\text { DETRATAMIENTO }\end{array}$ & B & S.E. & Wald & Df & Sig & $\mathrm{R}$ & $\operatorname{Exp}(B)$ \\
\hline PROGRAMA & & & 40,7705 & 5 & ,0000 & 2660 & \\
\hline PROGRAMA (1) & $-2,8289$ & 6144 & 21,2027 & 1 &, 0000 &,- 2101 & ,0591 \\
\hline PROGRAMA (2) & $-1,8190$ &, 5404 & 11,3318 & 1 & ,0008 &,- 1465 & , 1622 \\
\hline PROGRAMA (3) & $-2,1202$ & ,5866 & 13,0640 & 1 &, 0003 &,- 1595 & , 1200 \\
\hline PROGRAMA (4) & $-3,1127$ &, 5237 & 35,3238 & 1 &, 0000 & - 2768 &, 0445 \\
\hline PROGRAMA (5) &, 5461 & 1,0680 & ,2614 & 1 & 6091 & 6091 & 1,7264 \\
\hline
\end{tabular}

Gráfica 1

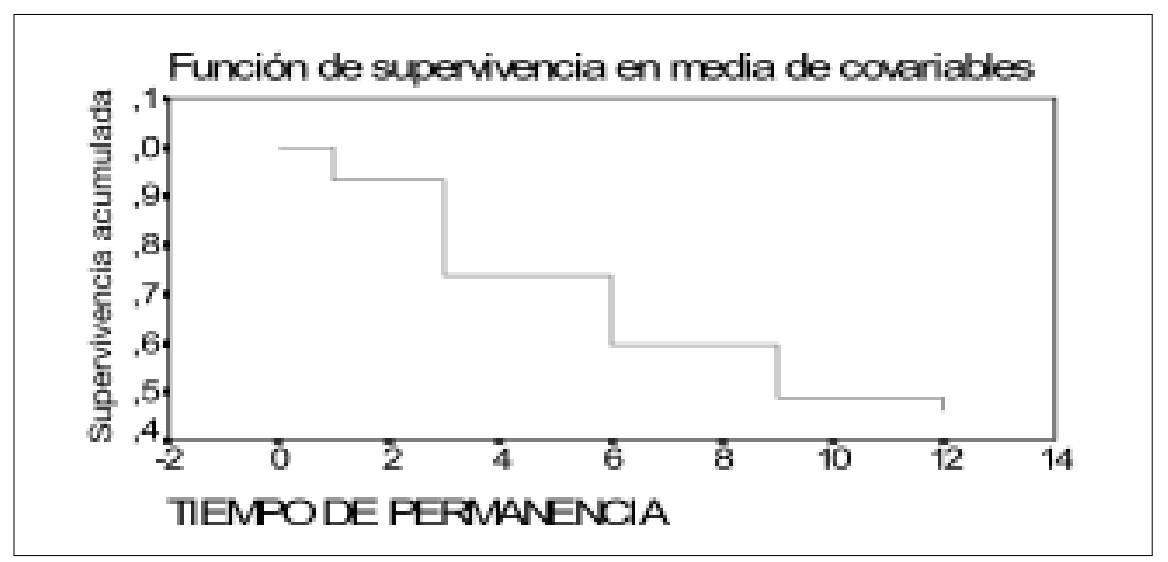

tanto a los tres como a los seis meses $(73.2 \%$ y $59 \%$ respectivamente) manteniéndose la adherencia a los 9 meses y disminuyendo en menor medida hasta un $48.9 \%$ a los 12 meses. En la tabla IV se presentan los resultados de la adherencia al tratamiento. En este sentido, observamos cómo estudios recientes sobre la retención en los tratamientos, apuntan hacia una alta tasa de abandonos (del 48 al $80 \%$ según el tipo de programa y el corte temporal) al margen del abordaje utilizado para ello: programas ambulatorios o programas residenciales (Apocada, et al., 1995; Arias et al. 1996; Secades et al., 1996; Secades et al. 1998).

\section{Correlación entre variables:}

Al cruzar las diferentes variables estudiadas con la variable "adherencia al tratamiento", observamos que las variables "edad" y "tipo de programa de tratamiento" correlacionan significativamente con adherencia en todos los cortes temporales evaluados. A mayor edad mayor probabilidad de continuar en tratamiento y asimismo, aquellos sujetos que realizan un programa de mantenimiento con antagonistas o con metadona presentan una probabilidad de adherencia mayor que en el resto de programas. Muchos estudios apuntan hacia mejores resultados en programa de mante- 


\begin{tabular}{|c|c|c|c|c|c|c|c|c|}
\hline \multicolumn{9}{|c|}{ TABLA VII - RESULTADOS DE LA REGRESIÓN DE COX SEGÚN LOS GRUPOS DE VARIABLES } \\
\hline VARIABLES & Variables seleccionadas & B & S.E. & Wald & $d f$ & Sig & $\mathrm{R}$ & $\operatorname{Exp}(B)$ \\
\hline \multirow[t]{6}{*}{ DEMOGRÁFICAS } & SITUACIÓN LABORAL & & & 11,864 & 4 & ,018 & 051 & \\
\hline & SIT.LAB. (1) & $-2,25$ & ,741 & 9,25 & 1 &, 002 &,- 070 & 104 \\
\hline & SIT. LAB. (2) & $-3,12$ & 1,021 & 9,376 & 1 & ,002 &,- 070 & ,043 \\
\hline & SIT. LAB. (3) & $-2,33$ & 1,238 & 3,549 & 1 & 059 &,- 032 & 097 \\
\hline & SIT. LAB. (4) & $-2,37$ & ,734 & 10,458 & 1 &, 001 &,- 076 & ,093 \\
\hline & EDAD &,- 267 &, 098 & 7,398 & 1 & ,006 &,- 060 &, 765 \\
\hline \multirow[t]{9}{*}{ PSICOSOCIALES } & ACOMPAÑADO POR & & & 13,446 & 8 & ,097 &, 000 & \\
\hline & ACOMP. POR (1) & $-11,96$ & 426,54 &, 000 & 1 & ,977 &, 000 & 6,373 \\
\hline & ACOMP. POR (2) & 1,409 & ,478 & 8,665 & 1 & ,003 & 123 & 4,09 \\
\hline & ACOMP. POR (3) & ,752 & .578 & 1,694 & 1 & 193 & ,000 & 2,12 \\
\hline & ACOMP. POR (4) & 486 & ,401 & 1,472 & 1 &, 225 &, 000 & 1,62 \\
\hline & ACOMP. POR (5) & 1,348 & ,649 & 4,314 & 1 & 037 & ,072 & 3,85 \\
\hline & ACOMP. POR (6) &,- 284 & 1,041 &, 074 & 1 &, 784 &, 000 &, 75 \\
\hline & ACOMP. POR (7) &, 528 & 1,042 & 257 & 1 & 611 &, 000 & 1,69 \\
\hline & ACOMP. POR (8) &, 005 &, 456 &, 000 & 1 & 991 &, 000 & 1,00 \\
\hline \multirow[t]{5}{*}{ ESTADIO DE CAMBIO } & ESTADIO & & & 11,895 & 4 & ,018 & ,051 & \\
\hline & ESTADIO(1) & 661 &, 387 & 2,911 & 1 & 088 & .024 & 1,93 \\
\hline & ESTADIO(2) &,- 217 &, 250 &, 753 & 1 &, 385 &, 000 & 80 \\
\hline & ESTADIO(3) & ,023 & 1,024 &, 000 & 1 & 981 & ,000 & 1,02 \\
\hline & ESTADIO(4) & 418 & ,309 & 1,829 & 1 & ,176 & ,000 & 1,51 \\
\hline
\end{tabular}

nimiento con metadona (PMM) y Comunidades Terapéuticas, frente a los llamados programas libres de drogas (Simpson, 1981, 1997; De Leon, 1984). Otros estudios obtienen que las tasas de abandonos más reducidas, se encuentran en los programas de desintoxicación (hospitalarias y ambulatorias) y en los PMM, concretamente en aquellos de baja exigencia se comprueban tasas de retención a los 2 años del $72 \%$ (Torrens, M. Et al., 1996).

Los sujetos que han seguido "tratamientos previos" presentaron una adherencia mayor a los 6,9 y 12 meses; y el resto de los intervalos medidos ( 1 y 3 meses) presentaron una relación muy cercana a la significación estadística $\quad(p=0.058$ al mes y $p=0.056$ a los 3 meses). El estadio de cambio predice adherencia al tratamiento al mes y a los 3 meses en el sentido de que los sujetos que se ubicaban en precontemplación y actuación tienen una probabilidad menor de continuar en tratamiento que los contempladores y los preparados para la acción. Los que acuden solos, acompañados por ambos padres o con un amigo, tienen mayor probabilidad de continuar tratamiento a los 9 y 12 meses. Por último, encontramos que la adherencia al mes correlaciona significativamente con la adherencia a los tres, seis, nueve y doce meses, lo cual indica que el hecho de no abandonar durante el primer mes supone una adherencia mayor en los cortes temporales posteriores medidos.

\section{MODELO DE SUPERVIVENCIA}

Se eligió la regresión de Cox para estudiar la relación entre el tiempo de permanencia en tratamiento y el resto de las variables estudiadas (variables independientes). Para la selección de variables se empleó el método Forward. Se comprobó la bondad del ajuste, es decir cuán probables eran los resultados muestrales a partir del modelo ajustado, también denominado verosimilitud, estimando una buena bondad de ajuste rechazar la hipótesis nula al nivel de significación del 0.05 .

En primer lugar se incluyeron todas las variables estudiadas dando como resultado que el tipo de tratamiento que realiza el sujeto influye en el tiempo de permanencia en el mismo (ver tabla VI). Para un sujeto en particular, si los valores de la variable "tipo de tratamiento" se desvían poco respecto de los valores medios, la función de supervivencia correspondiente será parecida a la curva analizada, estimándose que la probabilidad de abandono disminuirá de forma muy acusada a partir de los 3 meses (ver gráfica 1).

\section{Gráfica 1}

Como hemos señalado anteriormente, el tipo de tratamiento ya demostraba su significación estadística en todos los cortes temporales, por lo que este resultado incidiría en la importancia que el programa de tratamiento tiene en el tiempo de permanencia. Los programas de mantenimiento con metadona son los que 
presentan mayor tasa de adherencia, seguidos de los programas de mantenimiento con antagonistas y finalmente los programas libres de drogas. En esta línea Simpson et al. (1997), López et al. (1996) encuentran resultados similares.

Un segundo paso en el análisis estadístico fue realizar nuevas regresiones de Cox con cada uno de los grupos de variables por separado: demográficas, toxicológicas y psicosociales. En la tabla VII se resumen las variables seleccionadas de cada uno de los grupos.

\section{TABLA VII}

De todas las variables demográficas fueron seleccionadas la edad y la situación laboral; de las variables psicosociales la forma de acudir al centro (solo, acompañado por...) y finalmente, la variable estadio de cambio también fue seleccionada por la prueba estadística. En todos los cortes temporales medidos, una menor edad se asocia a un mayor abandono y contrariamente a mayor edad mayor permanencia. En cuanto a la situación laboral, señalar que aquellos sujetos que están trabajando abandonan más que los que se encuentran en situación de parados. Por otro lado, los sujetos que acuden acompañados por la madre, ambos padres o varios familiares son los que más abandonan, mientras que los acompañados por pareja no consumidora, padre o hermanos presentan mayor probabilidad de retención. Finalmente, los sujetos preparados para la acción y los contempladores son los que menos abandonan, frente a los precontempladores, y en menor medida los que se ubican en el estadio de actuación, que abandonan en una proporción estadísticamente mayor.

\section{DISCUSIÓN}

En lo referente a los estadios de cambio encontramos que la gran mayoría de sujetos cuando acuden a los Centros asistenciales en demanda de tratamiento, no se plantean seriamente un abandono a corto plazo del uso de drogas y el cambio de su estilo de vida, y que la "demanda" obedecería mas a presiones externas que a un deseo real de cambio. Por lo tanto, el tipo de intervención más adecuado consistirá en ayudarles a progresar en los diferentes estadios de cambio, más que a la consecución de la abstinencia, al menos a corto plazo.

La mayor parte de los abandonos se producen durante los 6 primeros meses de tratamiento, apareciendo una tendencia a la retención a partir de ese momento. Podemos interpretar que, en la medida en que el sujeto va adquiriendo compromiso con el tratamiento, consolidándose una buena alianza terapéutica con el/los profesionales y desarrollando una mayor percepción de autoeficacia, disminuye considerablemente la probabilidad de abandono. Ya en la década de los 70, Vallant (1977) y Simpson (1979) concluyen que un indicador de buen pronóstico en el tratamiento es la permanencia de los pacientes en programa por encima de los 3 meses. Asimismo, diferentes estudios realizados en programas residenciales encuentran que la mayor parte de pacientes que abandonan lo hacen durante los 3 primeros meses de tratamiento (Kooyman, 1996; Santos, P. et al., 1998).

Diversos estudios apuntan hacia la tasa de retención como la variable más importante para predecir los resultados de la intervención terapéutica (Estudio EMETYST y Programa DROSS). En esta línea, algunos estudios han demostrado que un indicador de buen pronóstico en el tratamiento es la permanencia de los pacientes en programa por encima de los tres meses, Palomares et al. (1998), Simpson (1979, 1981), Vaillant (1973), Sirotnick et al. (1978).

La variable estadio de cambio puede ser una buena predictora de la adherencia a corto plazo (durante los 3 primeros meses). Las diferencias encontradas, vendrían explicadas por la alta tasa de retención que presentan los sujetos preparados para la acción, frente a los precontempladores que abandonan en una proporción estadísticamente mayor. Por otro lado, queremos resaltar la alta tasa de abandono de los usuarios ubicados en el estadio de actuación, siendo los que más abandonan después de los precontempladores (al mes y tres meses). Las posibles explicaciones las podríamos hallar atendiendo a alguna/s de las siguientes cuestiones: un error en la ubicación en el estadio a partir de una información verbalizada por el sujeto no ajustada a la realidad, y/o considerar que no necesita continuar un tratamiento ya que se considera capaz de mantener la abstinencia, y/o acudir a tratamiento presionado o a petición de alguna institución, y/o producirse una recaída.

A la vista de los resultados obtenidos no podemos concluir que el estadio de cambio sea una potente variable predictora de la adherencia al tratamiento, ya que solamente se muestra estadísticamente significativa al mes y a los 3 meses, mientras que otras variables estudiadas sí predicen adherencia durante todos los cortes temporales medidos (edad, tipo de tratamiento que inicia), y en menor medida (tratamientos anteriores, tiempo máximo de abstinencia previa y el tipo de acompañamiento) que tienden a predecir adherencia en los últimos cortes temporales. Esto podría ser debido a que el estadio de cambio es una variable dinámica por lo que es lógico que presente escasa capacidad predictiva, pero $\quad-y$ esto es lo positivo- es susceptible de intervención y modificación. 
AGRADECIMIENTOS: La presente investigación ha sido becada por la Dirección General de Drogodependencias de la Generalitat Valenciana.

\section{BIBLIOGRAFÍA}

ABELLANAS, L. y MC. LELLAN, A.T. (1993): Stage of chage by drug problem in concurrent opioid, cocaine, and cigarette users. Journal of Psychoactive Drugs, 25, 307-313.

ALBIACH, C.; Llinares Pellicer, M.C., Santos Diez, P., Palau Muñoz, C. (1996): Estudio descriptivo sobre sujetos heroinómanos que demandan asistencia en servicios ambulatorios: evaluación de los estadios de cambio. En II Jornadas sobre drogodependencias. Ed.: Salvador Amigo. Universidad Politécnica de Valencia.

APOCADA, I. et al. (1995): Características de toxicómanos que no inician e interrumpen el tratamiento. Adicciones, 2, 159-167.

ARIAS, F. et al. (1996): Predictores evolutivos en un Programa de mantenimiento con naltrexona. Adicciones, vol. 8 (4), 479-501.

ARIAS, F.; LOPEZ, J.J.; OCHOA, E. (1996): Predictores evolutivos en un programa de mantenimiento con naltrexona. Adicciones, vol. 8 (4), 479-500.

BECOÑA, E. (1994): Evaluación de la conducta de fumar. En J.L. Graña (ED.), Conductas adictivas. Teoría, evaluación y tratamiento (pp. 403-545). Madrid. Debate.

CABALlERO, L., MATIA, J.; PEDERNAL, E. (1988): Dos años de experiencia del registro acumulativo de drogodependientes de Madrid. Comunidad Drog. $\mathrm{N}^{\circ}$ 8: $37-46$.

CUSKER C.G., BROWN K. (1996): Cue-responsivity phenomenon in dependent drinkers: "personality" vulnerability and anxiety as intervening variables. $\mathrm{Br}$. J. Addict. V. 86; $n^{\circ}$ 7: 905-912.

DE LEON, G. (1984): Program-based evaluation research in therapeutic communities. En F.M. Tims y J.P. Ludford (eds). Ealuation of Drug Treatment Programs (pp. 7-29). New York. The Naworth.

DICLEMENTE, C.C.; PROCHASKA, J.O. (1985): Processes and stages of chage: coping and competence in smoking behavior change. In Shiffman and T.A. Wills (Eds) Coping and substance abuse (319-343). San Diego C.A. Academic Press.

FARKAS, A.J. (1996): Is stage of change a useful measure of the likelihood of smoking cessation? Annal of Behavioral Medicine. Vol. 18 (2): 79-86.

FERNÁNDEZ, C., et al. (1996): Características de personalidad de heroinómanos tratados en una Comunidad Terapéutica. Adicciones, 8 (1), 33-51.

GUTIERREZ, E.; SAIZ, P.; GONZÁLEZ, P.; FERNÁNDEZ, j.; BOBES, J. (1998): Trastornos de personalidad en adictos a opiáceos en tratamiento con agonistas vs. antagonistas. Adicciones, 10 (2), 121-129.

HUBBARD et al. (1984): Treatment outcome prospective study (tops): Client characteristics and behaviors before, during and after treatment. En: Drug abuse treatment evaluation: strategies, progress and prospects. National Institute on drug abuse.

ISENHART, C.E. (1994): Motivational subtypes in an inpatient sample of substance abusers. Addictive Behaviors, 19, 463-475.

KOOYMAN, M. (1996): La Comunidad Terapéutica para drogodependientes. Bilbao. Mensajero.

LOPEZ N., MARCOS C., RUIZ R., CASETE L., CARRERA I.H., MARIÑO A. (1996): Estudio comparativo de la evolución a 12 meses en programa de mantenimiento de metadona versus programa de mantenimiento con naltrexona. Libro de Actas XXIII Jornadas Nacionales de Socidrogalcohol.

MARINA, P.; VAZQUEZ, J.; JIMENEZ, J. (1996): Los beneficios de la abstinencia: un estudio de seguimiento de adictos a la heroína. Adicciones, 8 (3), 295-309.

ORDOÑEZ, F., et al. (1995): Entorno y Conducta del drogodependiente ADVP-SIDA. Estudio en una muestra asturiana. Adicciones, 7 (3), 329-341.

PASTOR, M.A.; SANTOS, P.; PALOMARES, J.V. (1999): U.D.R. Balsa Blanca: estudio de una muestra de drogodependientes en programa de deshabituación. CD-ROM VI Encuentro Nacional sobre drogodependencias y su enfoque comunitario.

PALOMARES, J.V.; PASTOR, M.A.; SANTOS, P. (1998): La comunidad terapéutica: variables predictoras de evolución". CD-ROM II Congreso Iberoamericano de Psicología. 1998.

PROCHASKA et al. (1992a): In search of how people change: applications to addictive behaviors. American Psychologist, 47, 1102-1114.

PROCHASKA, J.O. et al. (1992b): Attendance and outcome in work site weight control program: processes and stages of change as process and predictor variables. Addictive Behaviors, 17, 35-45.

PROCHASKA, J.O. y VELICER, W. (1996): On models, methods and premature conclusions. Addiction, 91 (9), 1281-1292.

SANTOS, P.; PALOMARES, J.V.; PASTOR, M. A. (1997): Variables predictoras de la evolución de los sujetos en la Comunidad Terapéutica "Balsa Blanca". XIV Jornadas Nacionales de Socidrogalcohol. Libro de Actas.

SECADES, et al. (1991): Estudio de los determinantes en las recaídas de los heroinómanos. Revista Española de Drogodependencias, 16 (4), 285-293.

SECADES, U.; MENÉNDEZ, C. (1996): Retención de heroinómanos en un Centro de Día. XXIII Jornadas Nacionales de Socidrogalcohol. Oviedo. Libro de Actas.

SECADES V. et al (1998): Factores asociados a la retención de heroinómanos en un programa libre de drogas. Adicciones. Vol. 10, $n^{\circ} 1,53-59$. 
SIMPSON, D. (1979): The relation of time spent in drug abuse treatment to posttreatment outcome. Am. J. Psychiatry, 136, 1449-1453.

SIMPSON, D. (1981): Treatment for drug abuse: follow-up outcomes and lengh of time spent. Arch. Gen. Psychiatry, 38, 875-880

SIMPSON, D.; Jose, G.W.; Rowan-Szal, G.A. (1997): Drug abuse treatment retention and process effects on flollow-up outcomes. Drug and Alcohol Dependence. Vol 47 (3), 227-235.

SIROTINIK, K.A.; ROFFE, M.W. (1978): An investigation of the feasibility of predicting outcome indices in the treatment of heroin addiction. Int. J. Of the addictions, 13(1), 184-190.

TEJERO, A. y TRUJOLS, J. (1994): El modelo transteórico de Prochaska y DiClemente: un modelo denámico del cambio en el ámbito de las conductas adictivas.
En J.L. Graña (Ed) Conductas Adictivas: teoría, evaluación y tratamiento, 1-43. Madrid. Debate.

TORRENS, M. et al. (1996): Programas de metadona de bajo umbral. En: M. Casas, M. Gutiérrez y L. San (coord.). Avances en Drogodependencias. Monografías de Toxicomanías. Barcelona. Citran. Ediciones en Neurociencias. 169-205.

TUCFER et al. (1985): Determinants of substance abuse relapse. En M. Galizzo and S.A. Maisto (eds). Determinants of substance abuse: Biological, Psuchological an Environmental factors. New York: Plenum, 383-421.

VAILLANT, G.E. (1973): A twenty year follow-up of New York narcotic addicts. Arch. Gen. Psychiatry, 29, 237241.

VELICER, W.F. et al. (1995): An Empirical typology of subjets within stage of change. Addictive Behaviors 